\title{
Sum of Two Dimensional Fibonacci Sequence by Solutions of Second Order Difference Equations
}

\author{
G. B. A. Xavier, S. U. V. Kumar, B. Mohan
}

\begin{abstract}
In this paper, we introduce two dimensional difference operator and its inverse by which we obtain two dimensional Fibonacci sequence and its sum. Some theorems and interesting results on the sum of the terms of two dimensional Fibonacci sequence are derived. Suitable examples are provided to illustrate our results.
\end{abstract}

Keywords: Two dimensional difference operator, Two dimensional Fibonacci sequence, Closed form solution, Fibonacci summation formula.

\section{Introduction}

In 1984, Jerzy Popenda [5] introduced a particular type of difference operator on $u(k)$ as $\Delta_{\alpha} u(k)=u(k+1)-\alpha u(k)$. In 1989, Miller and Rose [8] introduced the discrete analogue of the Riemann-Liouville fractional derivative and its inverse $\Delta_{h}^{-v} f(t)([1,4])$. The sum of $m^{\text {th }}$ partial sums of products of higher powers of arithmetic and geometric progressions are derived in [9] by replacing $h$ by $\ell, v$ by $m$ and $f(t)$ by $u(k)$ in $\Delta_{h}^{-v} f(t)$.

In 2011, M.Maria Susai Manuel, et.al, [7] extended the operator $\Delta_{\alpha}$ to generalized $\alpha$-difference operator as $\underset{\alpha(\ell)}{\Delta} v(k)=v(k+\ell)-\alpha v(k)$ for the real valued function $v(k)$. In 2014, G.Britto Antony Xavier, et.al, [2] introduced $q$-difference operator as $\Delta_{q} v(k)=$ $v(q k)-v(k), q \in(0, \infty)$ and obtained finite series solution to the corresponding generalized $q$-difference equation $\Delta_{q} v(k)=u(k)$.

\section{Two Dimensional Fibonacci Sequence and Difference Operator}

Fibonacci and Lucas numbers cover a wide range of interest in modern mathematics as they appear in the comprehensive works of Koshy [6] and Vajda [10]. The $k$-Fibonacci

Manuscript received April 12, 2016; accepted July 1, 2016.

G. B. A. Xavier, S. U. V. Kumar, B. Mohan are with the Department of Mathematics,Sacred Heart College, Tirupattur - 635601, Vellore District Tamil Nadu, S. India 
sequence introduced by Falcon and Plaza [3] depends only on one integer parameter $k$ and is defined as

$$
F_{k, 0}=0, \quad F_{k, 1}=1 \quad \text { and } \quad F_{k, n+1}=k F_{k, n}+F_{k, n-1}, \quad \text { where } \quad n \geq 1, k \geq 1 .
$$

In particular, if $k=2$, the Pell sequence is obtained as

$P_{0}=0, \quad P_{1}=1 \quad$ and $P_{n+1}=2 P_{n}+P_{n-1}$ for $n \geq 1$.

Here we introduce two dimensional difference operator $\underset{\left(a_{1}, a_{2}\right) \ell}{\Delta} v(k)=v(k)-a_{1} v(k-$ $1)-a_{2} v(k-2)$ which generates two dimensional Fibonacci sequence and its sum.

Definition 1 For each pair $\left(a_{1}, a_{2}\right) \in \mathbb{R}^{2}$, where $\mathbb{R}$ is the set of all real numbers, a two dimensional Fibonacci sequence is defined as

$$
\underset{\left(a_{1}, a_{2}\right)}{F}(0)=1, \underset{\left(a_{1}, a_{2}\right)}{F}(1)=a_{1}, \underset{\left(a_{1}, a_{2}\right)}{F}(n)=a_{1} \underset{\left(a_{1}, a_{2}\right)}{F}(n-1)+a_{2} \underset{\left(a_{1}, a_{2}\right)}{F}(n-2), n \geq 2
$$

The sequence (1) becomes the well known Fibonacci sequence when $a_{1}=1=a_{2}$.

Example 1 (i) Taking $a_{1}=3$ and $a_{2}=4$ in (1), we get a two dimensioanl Fibonacci sequence $\{1,3,13,51,205,819, \cdots\}$.

(ii) When $a_{1}=0.5$ and $a_{2}=0.8$ in (1), we have a two dimensioanl Fibonacci sequence $\{1,0.5,1.05,0.925,1.3025,1.39125,1.737625, \cdots\}$.

(iii) In particular, $\underset{(2,1)}{F}(0)=0, \underset{(2,1)}{F}(1)=1$ and $\underset{(2,1)}{F}(n)=2 \underset{(2,1)}{F}(n-1)+\underset{(2,1)}{F}(n-2)$ for $n \geq 2$ gives the Pell sequence $\{0,1,2,5,12,29,70, \ldots\}$.

Similarly, one can obtain two dimensional Fibonacci sequences corresponding to each pair $\left(a_{1}, a_{2}\right) \in \mathbb{R}^{2}$.

Definition 2 For each pair $\left(a_{1}, a_{2}\right) \in \mathbb{R}^{2}$, a two dimensional difference operator on $v(k)$, denoted as $\underset{\left(a_{1}, a_{2}\right)}{\Delta} v(k)$, is defined as

$$
\underset{\left(a_{1}, a_{2}\right)}{\Delta} v(k)=v(k)-a_{1} v(k-1)-a_{2} v(k-2), \quad k \in[0, \infty),
$$

and its inverse is defined as below;

$$
\text { if } \underset{\left(a_{1}, a_{2}\right)}{\Delta} v(k)=u(k), \quad \text { then we write } \quad v(k)=\underset{\left(a_{1}, a_{2}\right)}{\Delta} u(k) .
$$

Lemma 1 Let $k \in(-\infty, \infty)$. If $1-\frac{a_{1}}{a}-\frac{a_{2}}{a^{2}} \neq 0$, then we have

$$
\underset{\left(a_{1}, a_{2}\right)}{\Delta} a^{k}=\frac{a^{k}}{1-\frac{a_{1}}{a}-\frac{a_{2}}{a^{2}}} .
$$


Sum of Two Dimensional Fibonacci Sequence by Solutions of Second Order Difference Equations 133

Proof Taking $u(k)=a^{k}$ in (2), we have $\underset{\left(a_{1}, a_{2}\right)}{\Delta} a^{k}=a^{k}\left[1-\frac{a_{1}}{a}-\frac{a_{2}}{a^{2}}\right]$

Now (4) follows from (3).

Theorem 1 (Two dimensional Fibonacci Summation Formula) Let $\underset{\left(a_{1}, a_{2}\right)}{F}(n)$ be the two dimensional Fibonacci sequence given in (1) and $v(k)$ be a solution to the second order difference equation $\underset{\left(a_{1}, a_{2}\right)}{\Delta} v(k)=u(k), k \in[0, \infty)$. Then we have

$$
v(k)-\underset{\left(a_{1}, a_{2}\right)}{F}(n+1) v(k-(n+1))-a_{2} \underset{\left(a_{1}, a_{2}\right)}{F}(n) v(k-(n+2))=\sum_{i=0}^{n} \underset{\left(a_{1}, a_{2}\right)}{F}(i) u(k-i)
$$

Proof For simplicity, we denote $\underset{\left(a_{1}, a_{2}\right)}{F}(n)$ by $F_{n}$ in this proof.

From (2) and (3), we arrive

$$
v(k)=u(k)+a_{1} v(k-1)+a_{2} v(k-2) .
$$

Replacing $k$ by $k-1$ and then substituting the value of $v(k-1)$ in (6), we get

$$
v(k)=u(k)+a_{1} u(k-1)+\left(a_{1}^{2}+a_{2}\right) v(k-2)+a_{1} a_{2} v(k-3)
$$

which gives

$$
v(k)=F_{0} u(k)+F_{1} u(k-1)+F_{2} v(k-2)+a_{2} F_{1} v(k-3),
$$

where $F_{0}, F_{1}$ and $F_{2}$ are given in (1).

Replacing $k$ by $k-2$ in (6) and then substituting $v(k-2)$ in (8), we obtain $v(k)=F_{0} u(k)+F_{1} u(k-1)+F_{2} u(k-2)+F_{3} v(k-3)+a_{2} F_{2} v(k-4)$, where $F_{3}$ is given in (1).

Repeating this process again and again, we get (5).

Corollary 1 Let $k \in(-\infty, \infty)$ and $1 \neq \frac{a_{1}}{a}-\frac{a_{2}}{a^{2}}$. Then we have

$$
\frac{a^{k}-\underset{\left(a_{1}, a_{2}\right)}{F}(n+1) a^{k-n-1}-a_{2} \underset{\left(a_{1}, a_{2}\right)}{F}(n) a^{k-n-2}}{1-\frac{a_{1}}{a}-\frac{a_{2}}{a^{2}}}=\sum_{i=0}^{n} \underset{\left(a_{1}, a_{2}\right)}{F}(i) a^{k-i} .
$$

Proof The proof of (9) follows by taking $u(k)=a^{k}$ and applying (4) in (5). 
The following example is an verification of (9).

Example 2 Taking $k=11, a=5, a_{1}=5, a_{2}=7$ and $n=4$ in (9), we get

$$
-\frac{25}{7}\left[5^{11}-\underset{(5,7)}{F}(5) 5^{6}-7 \underset{(5,7)}{F}(4) 5^{5}\right]=\sum_{i=0(5,7)}^{4} \underset{F}{F}(i) 5^{(11-i)}=330000000 .
$$

Corollary 2 (Fibonacci Summation Formula) For $n \in \mathbb{N}(0)=\{0,1,2,3, \ldots\}$, if $F_{n}$ denotes the $n^{\text {th }}$ term of the Fibonacci sequence, then we have

$$
F_{n+1}+F_{n}-1=\sum_{i=0}^{n} F_{i}
$$

Proof The proof follows by taking $a_{1}=a_{2}=a=1$ in (9).

The following example illustrates Fibonacci summation formula (10).

Example 3 Taking $n=20$ in (10), since $F_{21}=10946, F_{20}=6765$, we find $\sum_{i=0}^{20} F_{i}=F_{21}+F_{20}-1=17710$.

Theorem 2 Let $m \in \mathbb{N}(0)$ and $1-a_{1}-a_{2} \neq 0$. Then a closed form solution of the second order difference equation $v(k)-a_{1} v(k-1)-a_{2} v(k-2)=k^{m}$ is

$$
\underset{\left(a_{1}, a_{2}\right)}{\Delta} k^{m}=\frac{k^{m}}{1-a_{1}-a_{2}}+\sum_{i=1}^{m} \underset{\left(a_{1}, a_{2}\right)}{\Delta} \frac{(-1)^{i} m C_{i}\left(a_{1}+2^{i} a_{2}\right) k^{m-i}}{1-a_{1}-a_{2}}, \quad n \geq 1
$$

where $\underset{\left(a_{1}, a_{2}\right)}{\Delta} k^{0}=\frac{1}{1-a_{1}-a_{2}}$.

Proof Taking $v(k)=k^{0}$ in (2) and using (3), we get (12).

Taking $v(k)=\frac{k}{1-a_{1}-a_{2}}$ in (2), we get

$$
\underset{\left(a_{1}, a_{2}\right)}{\Delta} \frac{k}{\left(1-a_{1}-a_{2}\right)}=k+\frac{\left(a_{1}+2 a_{2}\right) k^{0}}{\left(1-a_{1}-a_{2}\right)} .
$$

Taking $v(k)=\frac{k^{2}}{1-a_{1}-a_{2}}$ in (2) gives

$$
\underset{\left(a_{1}, a_{2}\right)}{\Delta} \frac{k^{2}}{\left(1-a_{1}-a_{2}\right)}=k^{2}+\frac{2\left(a_{1}+2 a_{2}\right) k}{\left(1-a_{1}-a_{2}\right)}-\frac{\left(a_{1}+4 a_{2}\right) k^{0}}{\left(1-a_{1}-a_{2}\right)} .
$$


Sum of Two Dimensional Fibonacci Sequence by Solutions of Second Order Difference Equations 135

Similarly, taking $v(k)=\frac{k^{3}}{1-a_{1}-a_{2}}$ in (2), we obtain

$$
\begin{aligned}
\underset{\left(a_{1}, a_{2}\right)}{\Delta} \frac{k^{3}}{\left(1-a_{1}-a_{2}\right)} & =k^{3}+\frac{3\left(a_{1}+2 a_{2}\right) k^{2}}{\left(1-a_{1}-a_{2}\right)}-\frac{3\left(a_{1}+4 a_{2}\right) k}{\left(1-a_{1}-a_{2}\right)}+\frac{\left(a_{1}+8 a_{2}\right) k^{0}}{\left(1-a_{1}-a_{2}\right)} \\
& =k^{3}+\sum_{i=1}^{3} \frac{(-1)^{i+1} 3 C_{i}\left(a_{1}+2^{i} a_{2}\right) k^{3-i}}{1-a_{1}-a_{2}} .
\end{aligned}
$$

In general, we find that

$$
\underset{\left(a_{1}, a_{2}\right)}{\Delta} \frac{k^{m}}{\left(1-a_{1}-a_{2}\right)}=k^{m}+\sum_{i=1}^{m} \frac{(-1)^{i+1} m C_{i}\left(a_{1}+2^{i} a_{2}\right) k^{m-i}}{1-a_{1}-a_{2}} .
$$

The proof of (11) follows by applying $\underset{\left(a_{1}, a_{2}\right)}{\stackrel{-1}{\Delta}}$ on both sides of above and using (12).

Corollary 3 Taking $m=3$ in Theorem 2, we have

$$
\begin{array}{r}
\underset{\left(a_{1}, a_{2}\right)}{\Delta} k^{3}=\frac{k^{3}}{1-a_{1}-a_{2}}-\frac{3\left(a_{1}+2 a_{2}\right) k^{2}}{\left(1-a_{1}-a_{2}\right)^{2}}+\frac{6\left(a_{1}+2 a_{2}\right)^{2} k}{\left(1-a_{1}-a_{2}\right)^{3}}+\frac{3\left(a_{1}+4 a_{2}\right) k}{\left(1-a_{1}-a_{2}\right)^{2}} \\
-\frac{6\left(a_{1}+2 a_{2}\right)^{3}}{\left(1-a_{1}-a_{2}\right)^{4}}-\frac{6\left(a_{1}+2 a_{2}\right)\left(a_{1}+4 a_{2}\right)}{\left(1-a_{1}-a_{2}\right)^{3}}-\frac{\left(a_{1}+8 a_{2}\right)}{\left(1-a_{1}-a_{2}\right)^{2}},
\end{array}
$$

which is a closed form solution of the difference equation $\underset{\left(a_{1}, a_{2}\right)}{\Delta} v(k)=k^{3}$. Proof From (11), replacing $m=3$, we derive

$$
\begin{gathered}
\underset{\left(a_{1}, a_{2}\right)}{\Delta} k^{3}=\frac{k^{3}}{1-a_{1}-a_{2}}-\frac{3\left(a_{1}+2 a_{2}\right)}{\left(1-a_{1}-a_{2}\right)} \underset{\left(a_{1}, a_{2}\right)}{\Delta} k^{2}+\frac{3\left(a_{1}+4 a_{2}\right)}{\left(1-a_{1}-a_{2}\right)} \underset{\left(a_{1}, a_{2}\right)}{\Delta} k \\
-\frac{\left(a_{1}+8 a_{2}\right)}{\left(1-a_{1}-a_{2}\right)} \underset{\left(a_{1}, a_{2}\right)}{\stackrel{-1}{\Delta} k^{0} .}
\end{gathered}
$$

Applying (3) on the equations (13), (14) and using (12), we find

$$
\begin{aligned}
& \underset{\left(a_{1}, a_{2}\right)}{\stackrel{-1}{\Delta}} k=\frac{k}{1-a_{1}-a_{2}}-\frac{\left(a_{1}+2 a_{2}\right)}{\left(1-a_{1}-a_{2}\right)^{2}} \text { and hence } \\
& \underset{\left(a_{1}, a_{2}\right)}{\Delta} k^{2}=\frac{k^{2}}{1-a_{1}-a_{2}}-\frac{2\left(a_{1}+2 a_{2}\right) k}{\left(1-a_{1}-a_{2}\right)^{2}}+\frac{2\left(a_{1}+2 a_{2}\right)^{2}}{\left(1-a_{1}-a_{2}\right)^{3}}+\frac{\left(a_{1}+4 a_{2}\right)}{\left(1-a_{1}-a_{2}\right)^{2}} .
\end{aligned}
$$

Substituting the above values in (17) and using (12), we get (16). 
Corollary 4 If $v(k)=\underset{\left(a_{1}, a_{2}\right)}{\Delta} k^{m}$ is the closed form solution given in (11), then

$$
v(k)-\underset{\left(a_{1}, a_{2}\right)}{F}(n+1) v(k-(n+1))-a_{2} \underset{\left(a_{1}, a_{2}\right)}{F}(n) v(k-(n+2))=\sum_{i=0}^{n} \underset{\left(a_{1}, a_{2}\right)}{F}(i)(k-i)^{m} .
$$

Proof The proof follows by taking $u(k)=k^{m}$ in Theorem 1 .

Example 4 Let $k=7, m=3, n=4, a_{1}=3, a_{2}=4$ in Corollary (4). Then

$$
\sum_{i=0(3,4)}^{4} \underset{F}{F}(i)(7-i)^{3}=v(7)-\underset{(3,4)}{F}(5) v(2)-4 \underset{(3,4)}{F}(4) v(1)=11415 .
$$

The Fibonacci summation formula can also be obtained from (18).

Remark 1 Taking $a_{1}=a_{2}=1, m=0$ in (18) and using (12), we get the Fibonacci summation formula;

$$
F_{n+1}+F_{n}-1=\sum_{i=0}^{n} F_{i}
$$

Following theorem gives the inverse of two dimensional difference operator for product of two functions.

Theorem 3 Let $u(k)$ and $v(k)$ be two real valued functions. Then

$$
\begin{gathered}
\underset{\left(a_{1}, a_{2}\right)}{-1}[u(k) v(k)]=u(k) \underset{\left(a_{1}, a_{2}\right)}{\Delta} v(k)-a_{1} \underset{\left(a_{1}, a_{2}\right)}{\Delta}\left[\underset{\left(a_{1}, a_{2}\right)}{\Delta} v(k-1) \underset{(1,0)}{\Delta} u(k)\right] \\
-a_{2} \underset{\left(a_{1}, a_{2}\right)}{\Delta}\left[\underset{\left(a_{1}, a_{2}\right)}{\Delta} v(k-2) \underset{(0,1) \ell}{\Delta} u(k)\right] .
\end{gathered}
$$

Proof From (2), we arrive

$\underset{\left(a_{1}, a_{2}\right)}{\Delta}[u(k) w(k)]=u(k) w(k)-a_{1} u(k-1) w(k-1)-a_{2} u(k-2) w(k-2)$.

Adding and subtracting $a_{1} u(k) w(k-1), a_{2} u(k) w(k-2)$ on the right side, we obtain

$$
\underset{\left(a_{1}, a_{2}\right)}{\Delta}[u(k) w(k)]=u(k) \underset{\left(a_{1}, a_{2}\right)}{\Delta} w(k)+a_{1} w(k-1) \underset{(1,0)}{\Delta} u(k)+a_{2} w(k-2) \underset{(0,1)}{\Delta} u(k) .
$$

Taking $w(k)=\underset{\left(a_{1}, a_{2}\right)}{\stackrel{-1}{\Delta}} v(k)$ in the above and applying $\underset{\left(a_{1}, a_{2}\right)}{\stackrel{-1}{\Delta}}$ on both sides, we get the proof. 
Sum of Two Dimensional Fibonacci Sequence by Solutions of Second Order Difference Equations137

Corollary 5 A closed form solution of the generalized second order difference equation $v(k)-a_{1} v(k-1)-a_{2} v(k-2)=k^{2} a^{k}$ is given by

$$
\begin{aligned}
\underset{\left(a_{1}, a_{2}\right)}{-1} k^{2} a^{k}= & \frac{k^{2} a^{k}}{1-\frac{a_{1}}{a}-\frac{a_{2}}{a^{2}}}-\frac{2 a_{1} k a^{k-1}}{\left(1-\frac{a_{1}}{a}-\frac{a_{2}}{a^{2}}\right)^{2}}-\frac{4 a_{2} k a^{k-2}}{\left(1-\frac{a_{1}}{a}-\frac{a_{2}}{a^{2}}\right)^{2}} \\
& +\frac{a_{1} a^{k-1}}{\left(1-\frac{a_{1}}{a}-\frac{a_{2}}{a^{2}}\right)^{2}}+\frac{4 a_{2} a^{k-2}}{\left(1-\frac{a_{1}}{a}-\frac{a_{2}}{a^{2}}\right)^{2}}+\frac{2 a_{1}^{2} a^{k-2}}{\left(1-\frac{a_{1}}{a}-\frac{a_{2}}{a^{2}}\right)^{3}} \\
& +\frac{8 a_{1} a_{2} a^{k-3}}{\left(1-\frac{a_{1}}{a}-\frac{a_{2}}{a^{2}}\right)^{3}}+\frac{8 a_{2}^{2} a^{k-4}}{\left(1-\frac{a_{1}}{a}-\frac{a_{2}}{a^{2}}\right)^{3}} .
\end{aligned}
$$

Proof Taking $u(k)=k$ and $v(k)=a^{k}$ in (20), using (4) and (13), we find

$$
\underset{\left(a_{1}, a_{2}\right)}{\stackrel{-1}{\Delta}} k a^{k}=\frac{k a^{k}}{1-\frac{a_{1}}{a}-\frac{a_{2}}{a^{2}}}-\frac{a_{1} a^{k-1}}{\left(1-\frac{a_{1}}{a}-\frac{a_{2}}{a^{2}}\right)^{2}}-\frac{2 a_{2} a^{k-2}}{\left(1-\frac{a_{1}}{a}-\frac{a_{2}}{a^{2}}\right)^{2}} .
$$

Again, taking $u(k)=k^{2}$ and $v(k)=a^{k}$ in (20), using (4) and (14), we arrive

$$
\begin{gathered}
\underset{\left(a_{1}, a_{2}\right)}{-1} k^{2} a^{k}=\frac{k^{2} a^{k}}{1-\frac{a_{1}}{a}-\frac{a_{2}}{a^{2}}}-a_{1} \underset{\left(a_{1}, a_{2}\right)}{\Delta}\left[\left(\frac{a^{k-1}}{1-\frac{a_{1}}{a}-\frac{a_{2}}{a^{2}}}\right)(2 k-1)\right] \\
-a_{2} \underset{\left(a_{1}, a_{2}\right)}{\Delta}\left[\left(\frac{a^{k-2}}{1-\frac{a_{1}}{a}-\frac{a_{2}}{a^{2}}}\right)(4 k-4)\right] .
\end{gathered}
$$

Substituting (22) in the above and using (4), we get the proof of (21).

Corollary 6 If $v(k)=\underset{\left(a_{1}, a_{2}\right)}{\stackrel{-1}{\Delta}} k^{2} a^{k}$ is the closed form solution given in (21), then

$$
v(k)-\underset{\left(a_{1}, a_{2}\right)}{F}(n+1) v(k-(n+1))-a_{2} \underset{\left(a_{1}, a_{2}\right)}{F}(n) v(k-(n+2))=\sum_{i=0}^{n} \underset{\left(a_{1}, a_{2}\right)}{F}(i)(k-i)^{2} a^{k-i}
$$

Proof The proof follows by taking $u(k)=k^{2} a^{k}$ in Theorem 1 . 
Example 5 Let $k=6, n=4, a=4, a_{1}=8, a_{2}=16$ in Corollary (6). Then

$$
\sum_{i=0(8,16)}^{4} \underset{F}{F}(i)(6-i)^{2} 4^{6-i}=v(6)-\underset{(8,16)}{F}(5) v(1)-16 \underset{(8,16)}{F}(4) v(0)=1597440 .
$$

Conclusion: We obtained summation formula to two dimensional Fibonacci sequence by introducing two dimensional difference operator and have derived certain results on closed and summation form solution of second order difference equation which will be used to our further research. Further we will extend this definition to two dimensional generalized difference equation, which provides some applications in Digital Signal Processing and that is our future research.

\section{References}

[1] Bastos.N. R. O, Ferreira.R. A. C, And Torres.D. F. M., Discrete-Time Fractional Variational Problems, Signal Processing, 91(3)(2011),513-524.

[2] Britto Antony Xavier.G, Gerly.T.G and Nasira Begum.H, Finite Series of Polynomials and Polynomial Factorials arising from Generalized q-Difference operator, Far East Journal of Mathematical Sciences,94(1)(2014), 47-63.

[3] Falcon.S and Plaza.A, On the Fibonacci $k$-numbers, Chaos, Solitons and Fractals, vol.32, no.5, pp. 1615-1624, 2007.

[4] Ferreira.R. A. C AND Torres.D. F. M, Fractional h-difference equations arising from the calculus of variations, Applicable Analysis and Discrete Mathematics, 5(1) (2011), 110-121.

[5] Jerzy Popenda and Blazej Szmanda, On the Oscillation of Solutions of Certain Difference Equations, Demonstratio Mathematica, XVII(1), (1984), 153 - 164.

[6] Koshy.T, Fibonacci and Lucas Numbers with Applications, Wiley-Interscience, New York, NY, USA, 2001.

[7] Maria Susai Manuel.M, Chandrasekar.V and Britto Antony Xavier.G, Solutions and Applications of Certain Class of $\alpha$-Difference Equations, International Journal of Applied Mathematics, 24(6) (2011), 943-954.

[8] Miller.K.S AND Ross.B, Fractional Difference Calculus in Univalent Functions, Horwood, Chichester, UK, (1989),139-152.

[9] Susai Manuel.M, Britto Antony Xavier.G, Chandrasekar.V and PuGalarasu.R, Theory and application of the Generalized Difference Operator of the $n^{\text {th }}$ kind(Part I), Demonstratio Mathematica, 45(1)(2012), 95-106.

[10] VAJDA.S, Fibonacci and Lucas Numbers, and the Golden Section, Ellis Horwood, Chichester, UK, 1989. 